\title{
Correlation of intestinal microbiota with overweight and obesity in Kazakh school children
}

Peiru $\mathrm{Xu}^{1,2^{*+}}, \mathrm{Min} \mathrm{Li}^{1,2+}$, Jihong Zhang ${ }^{1}$ and Tao Zhang ${ }^{3}$

\begin{abstract}
Background: This study sought to investigate a possible correlation between the intestinal microbiota, Bacteroidetes and Firmicutes, and obesity in Kazakh school children, aged 7-13 ( $n=175)$.

Results: Obese subjects had significantly greater systolic blood pressure, waist and hip circumference, as well as HOMA-IR as compared to normal and overweight participants. In addition, Bacteroides copy number and Bact/Firm ratios were significantly lower in the obese group as compared to the normal and overweight groups $(P<0.0167)$. This difference is only significant in girls, but not in boys when stratified by gender. Furthermore, a negative correlation between BMI and Bacteroidetes copy number $(r=-0.18, P=0.017)$ as well as Bact/Firm $(r=-0.22$, $P=0.003)$ was observed.

Conclusion: An association between reduced gut Bacteroidetes and Bact/Firm ratio with obesity in female Kazakh children was identified. Further studies are necessary to elucidate the mechanism behind these changes as well as the value of determining their presence for predicting obesity.
\end{abstract}

Keywords: Obesity, Bacteroidetes, Firmicutes, Kazakh

\section{Background}

The incidence of obesity is increasing in an exponential manner worldwide and cannot be explained by genetic factors alone. Thus, a potential role for environmental factors (e.g., life style, geographical environment, feeding patterns etc.) has been increasingly explored in the pathogenesis of obesity. Recent evidence has revealed the influence of gut microbiota on the regulation of nutrient absorption, metabolism, and immune response [1,2].

In vivo studies have demonstrated that an imbalance in gut microbiota might play an important role in the pathogenesis of obesity [3-7]. Specifically, Ley et al. [8] observed reduced Bacteroidetes and increased Firmicutes levels in obese $(\mathrm{ob} / \mathrm{ob})$ mice. However, the correlation between an imbalance in gut microbiota and obesity

\footnotetext{
* Correspondence: xupeiru126@126.com

${ }^{\dagger}$ Equal contributors

'Department of Pediatrics, First Affiliated Hospital of Xinjiang Medical University, 137 Liyushan South Road, Urumqi, Xinjiang Uyghur Autonomous Region 840054, People's Republic of China

${ }^{2}$ Xinjiang Evidence-based Medicine Research Institute, 137 Liyushan South Road, Urumqi, Xinjiang, Uyghur Autonomous Region 830054, People's Republic of China

Full list of author information is available at the end of the article
}

varies among different human populations. Whereas some studies have observed reduced Bacteroidetes in obese subjects $[4,6,9]$, others have reported opposite results [10,11]. In addition, Duncan et al. [12] found no marked difference in Bacteroidetes levels between obese and normal weight subjects.

Bacteroidetes are nonendospore-forming anaerobes with bile resistance, accounting for more than $25 \%$ of gastrointestinal microbiota [13-15]. Because they absorb and metabolize polysaccharides [3] as well as promote the absorption of monosaccharides $[16,17]$, their metabolic activities may be related to obesity occurrence [18]. In addition, Bacteroidetes help maintain the balance in gastrointestinal microbiota $[17,19]$. Although the compositions of gastrointestinal microbiota have been identified, the ways in which these bacteria function remain poorly understood.

Because many of the previous studies linking gut microbiota with obesity have been performed in small groups, which may account for the discrepant results reported, this case-controlled study sought to investigate the role of gut microbiota and obesity in Kazakh children with more participants. The Kazakh people represent a minority in the Xinjiang Province of China. Most 
Kazakhs live in farming communities and pastoral areas that are underdeveloped, and the incidence of overweight and obesity is relatively high [20,21]. Previous studies have confirmed that the occurrence of obesity in Kazakh preschool children was related to genetic factors $[22,23]$. In this study, real-time fluorescence quantitative PCR (Q-PCR) was employed to detect Bacteroidetes and Firmicutes levels and their possible correlation with obesity.

\section{Methods}

\section{Study participants and study design}

This case-controlled study was carried out in the Yili Kazakh Autonomous Prefecture of China. Kazakh children (ages 7-13 y) were recruited from 14 schools within two Counties (Yining and Altay Counties), 5 towns (Yining, Gongliu, Xinyuan, Burqin, and Fuyun) and three villages. Informed consent was obtained from the guardians for all study participants, and children were willing to participant in this study. This study was approved by the Ethics Committee of the First Affiliated Hospital of Xinjiang Medical University (Xinjiang, China).

The following exclusion criteria were applied to select the study participants: (1) children aged $<7$ y or $>13$ y; (2) use of antibiotics 2 weeks prior to fecal sample collection as they could alter the gastrointestinal microbiota [24]; (3) the presence of stress (e.g., trauma, severe infection, etc.) 2 weeks prior to fecal sample collection; (4) the presence of gastrointestinal symptoms, including abdominal pain, constipation or diarrhea; and (5) a polio vaccination within one month, which may alter gut microbiota levels by the induced immune response to the vaccine.

A total of 5360 children aged 7-13 y were invited to participate in the study. Fecal specimens were collected from 244 children; 69 subjects were excluded based on the exclusion criteria. Thus, analysis was performed in 175 children.

\section{Measurements and sample collection}

After physical examination, study participants meeting the inclusion criteria were recruited, and informed consent was obtained prior to initiation of the study. In the morning, fasting venous blood samples were collected from the participants by the nurses of the Department of Pediatrics. After incubation at room temperature for $30 \mathrm{~min}$, the serum was collected by centrifugation at $3000 \mathrm{r} / \mathrm{min}$ for $15 \mathrm{~min}$ and separated into aliquots to analyze fasting plasma glucose (FPG), lipid (triglyceride [TG], total cholesterol [TC], high density lipoprotein [HDL], low density lipoprotein [LDL]), and insulin levels using 7060 Automatic Analyzer (HITACHI, Tokyo,
Japan). Homeostasis model assessment of insulin resistance (HOMA-IR) was employed to evaluate the degree of insulin resistance [25] and calculated as follows: HOMA-IR $=($ FPG $\times$ FIN $) / 22.5$.

Body weight and height were measured, and body mass index (BMI) was calculated for each participant. Specifically, the children stood straight with their legs close together and arms hanging naturally. Hip circumference was measured along the greater trochanter (accuracy: $0.1 \mathrm{~cm}$ ). The criteria for overweight/obesity were developed by the Institute of Child and Adolescent Health of Beijing University for Chinese school-age children and adolescents according to BMI [26], which is specific for age and gender. As shown in Table 1, 84 were diagnosed with overweight/obesity (62 with overweight; 22 with obesity), and the mean age was $9.82 \pm$ $1.96 \mathrm{y}$, and 91 children had normal BMI with a mean age of $9.92 \pm 1.98 \mathrm{y}$.

Stool collection boxes were given to each study participant with instructions on proper collection. Fresh feces were collected in the early morning. In the event that the children did not defecate in the early morning, feces were collected at any time of the morning. After collection, the fecal specimens were sent to the physical examination room and stored at $-20^{\circ} \mathrm{C}$.

\section{Real-time quantitative PCR (Q-PCR)}

Total DNA was extracted from the gut microbiota isolated from the fecal samples. Specifically, the samples were thawed, and total DNA was extracted from 0.2$0.4 \mathrm{~g}$ of the feces using a rapid DNA extraction kit (Beijing BioTeke Corporation, Beijing, China). Isolated DNA was then stored at $-20^{\circ} \mathrm{C}$ until subsequent use in Q-PCR.

To prepare the DNA standards, a sequence with $483 \mathrm{bp}$ in length was prepared and inserted into the $\mathrm{PCR}^{\circledR}$-Blunt II TOPO ${ }^{\circledR}$ vector (Invitrogen, USA). To generate the standard curve, the absolute number of template was $10^{10} / \mu \mathrm{L}$. The following serial dilutions of the original solution were used to generate the standard curve: $10^{8} / \mu \mathrm{L}, 10^{7} / \mu \mathrm{L}, 10^{6} / \mu \mathrm{L}, 10^{5} / \mu \mathrm{L}, 10^{4} / \mu \mathrm{L}$ and $10^{3} /$ $\mu \mathrm{L}$. The standard curves were obtained using the $\mathrm{ABI}$ 7500 Fast Q-PCR detecting system (Applied Biosystem, USA) and 7000 System SDS Software for qPCR.

To determine the absolute number of Bacteroidetes and Firmicutes in the gut microbiota, primers and probes (Invitrogen, Grand Island, NY) for the conservative sequence of the $16 \mathrm{~S}$ rRNA genes of both strains were synthesized according to those described previously (Table 1) [27-31] along with the Platinum ${ }^{\circledR}$ Taq DNA polymerase (Invitrogen). PCR reactions were denatured at $95^{\circ} \mathrm{C}$ for $2 \mathrm{~min}$ followed by 45 cycles of $95^{\circ} \mathrm{C}$ for $15 \mathrm{~s}$ and $60^{\circ} \mathrm{C}$ for $1 \mathrm{~min}$. 
Table 1 Sequences of primers

\begin{tabular}{llll}
\hline Primer Name & Sequence $\left(\mathbf{5}^{\prime}-\mathbf{3}^{\prime}\right)$ & Tm $\left({ }^{\circ} \mathrm{C}\right)$ & Target length \\
\hline Firm-primer-F & GTCAGCTCGTGTCGTGA & $60^{\circ} \mathrm{C}$ \\
\hline Firm-primer-R & CCATTGTAKYACGTGTGT & $60^{\circ} \mathrm{C}$ \\
\hline Firm-probe & VIC-GTCAANTCATCATGCC-MGBNFQ & $65^{\circ} \mathrm{C}$ \\
\hline Bact-primer-F & AGCAGCCGCGGTAAT & $60^{\circ} \mathrm{C}$ \\
\hline Bact-primer-R & CTAHGCATTCACCGCTA & $60^{\circ} \mathrm{C}$ \\
\hline Bact-probe & FAM-CCCTTTAAACCC-MGBNFQ & $65^{\circ} \mathrm{C}$ \\
\hline
\end{tabular}

\section{Statistical analysis}

Data were presented as means \pm standard deviations (mean \pm SD) for continuous data and $\mathrm{n}(\%)$ for categorical data. Differences between groups were compared using one-way ANOVA with a post-hoc Bonferroni test for continuous data or Kruskal-Wallis with Mann-Whitney U test when the data were not normally distributed. The Pearson chi-square test was used for categorical data. Furthermore, Spearman's correlation analysis was used to identify the correlation of BMI with levels of Bacteroidetes and Firmicutes. All statistical assessments were two-tailed and $P$-values $<0.05$ were considered statistically significant. Statistical analyses were performed using SPSS 15.0 statistics software (SPSS Inc, Chicago, IL, USA).

\section{Results}

A total of 175 subjects (87 boys and 88 girls) with a mean age of 9.87 y $(S D=1.97)$ were enrolled for evaluation. Subjects were grouped into the normal, overweight, or obese groups based upon their BMI. As shown in Table 2, demographic information, clinical characteristics, and the presence of Bacteroidetes and Firmicutes are shown for each group. Among the groups, significant differences in BMI, SBP, DBP, waist and hip circumference, insulin, and HOMA-IR levels were noted (all $P<0.05$ ). Obese subjects had significantly greater SBP, waist and hip circumference, as well as HOMA-IR as compared to normal and overweight participants $(P<0.05)$. In addition, significant differences in

Table 2 Subjects' demographics, characteristics and microbe microbiota data by group

\begin{tabular}{|c|c|c|c|c|c|}
\hline Variables & Total & Normal group & Overweight group & Obesity group & P-values \\
\hline & $(n=175)$ & $(n=91)$ & $(n=62)$ & $(n=22)$ & \\
\hline Age (y) & $9.87 \pm 1.97$ & $9.92 \pm 1.98$ & $9.65 \pm 1.87$ & $10.32 \pm 2.19$ & 0.368 \\
\hline Sex & & & & & 0.906 \\
\hline Boys & $87(49.7)$ & $45(49.5)$ & $30(48.4)$ & $12(54.5)$ & \\
\hline Girls & $88(50.3)$ & $46(50.5)$ & $32(51.6)$ & $10(45.5)$ & \\
\hline $\mathrm{BMI}, \mathrm{Kg} / \mathrm{m}^{2}$ & $18.87 \pm 3.45$ & $16.53 \pm 1.69$ & $20.14 \pm 1.83^{\dagger}$ & $24.94 \pm 3.11^{1 \neq}$ & $<0.001^{*}$ \\
\hline $\mathrm{SBP}, \mathrm{mmHg}$ & $97.66 \pm 14.93$ & $94.06 \pm 12.68$ & $98.34 \pm 13.21$ & $110.64 \pm 20.45^{1 \neq}$ & $<0.001^{*}$ \\
\hline $\mathrm{DBP}, \mathrm{mmHg}$ & $62.16 \pm 9.15$ & $60.38 \pm 8.1$ & $63.07 \pm 9.15$ & $66.93 \pm 11.39^{\dagger}$ & $0.005^{*}$ \\
\hline Waist, cm & $63 \pm 8.7$ & $58.27 \pm 4.91$ & $65.08 \pm 6.75^{\dagger}$ & $76.72 \pm 9.22^{1 \neq}$ & $<0.001^{*}$ \\
\hline Hip, cm & $74.48 \pm 9.98$ & $70.26 \pm 6.65$ & $76.04 \pm 8.7^{\dagger}$ & $87.52 \pm 12.41^{\dagger \neq}$ & $<0.001^{*}$ \\
\hline $\mathrm{FPG}, \mathrm{mmol} / \mathrm{L}$ & $4.81 \pm 0.84$ & $4.88 \pm 1.03$ & $4.73 \pm 0.57$ & $4.8 \pm 0.61$ & 0.569 \\
\hline Triglyceride, mmol/L & $1.21 \pm 0.53$ & $1.14 \pm 0.47$ & $1.27 \pm 0.58$ & $1.36 \pm 0.55$ & 0.194 \\
\hline Cholesterol, mmol/L & $3.67 \pm 0.71$ & $3.73 \pm 0.71$ & $3.65 \pm 0.68$ & $3.52 \pm 0.81$ & 0.424 \\
\hline $\mathrm{HDL}, \mathrm{mmol} / \mathrm{L}$ & $1.38 \pm 0.51$ & $1.35 \pm 0.48$ & $1.38 \pm 0.56$ & $1.53 \pm 0.46$ & 0.206 \\
\hline $\mathrm{LDL}, \mathrm{mmol} / \mathrm{L}$ & $1.58 \pm 0.43$ & $1.57 \pm 0.45$ & $1.58 \pm 0.37$ & $1.62 \pm 0.48$ & 0.885 \\
\hline Insulin, mmol/L & $6.55 \pm 3.74$ & $6.1 \pm 3.47$ & $6.21 \pm 3.28$ & $9.29 \pm 4.86^{\ddagger}$ & $0.006^{*}$ \\
\hline HOMA-IR & $1.42 \pm 0.87$ & $1.34 \pm 0.83$ & $1.33 \pm 0.76$ & $1.99 \pm 1.14^{\dagger \neq}$ & $0.016^{*}$ \\
\hline Bacteroidetes $\times 10^{7}$ copies $/ \mu \mathrm{L}$ & $1.31 \pm 1.94$ & $1.5 \pm 2.2$ & $1.37 \pm 1.77$ & $0.33 \pm 0.47^{\dagger}$ & $0.002^{*}$ \\
\hline Firmicutes $\times 10^{7}$ copies $/ \mu \mathrm{L}$ & $2.58 \pm 4.52$ & $2.43 \pm 4.53$ & $2.05 \pm 3.01$ & $4.7 \pm 7.01$ & 0.628 \\
\hline Bact/Firm & $0.98 \pm 0.71$ & $1.06 \pm 0.62$ & $1.03 \pm 0.82$ & $0.48 \pm 0.52^{\dagger \neq}$ & $<0.001^{*}$ \\
\hline
\end{tabular}

$(\mathrm{N}=175)$.

Data were presented as mean \pm SD for continuous data and $n(\%)$ for categorical data.

Abbreviations: BMI, body mass index; SBP, systolic blood pressure; DBP, diastolic blood pressure; FPG, fasting plasma glucose; HDL, high density lipoprotein; LDL,

low density lipoprotein; Bact/Firm, ratio of Bacteroidetes to Firmicutes.

${ }^{*} P<0.05$, indicated significant differences among groups.

${ }^{t, \ddagger} P<0.0167$, indicated significant differences as compared with the ${ }^{\dagger}$ normal and ${ }^{\ddagger}$ overweight groups. 
DBP and Bacteroidetes were observed between the obese and normal groups.

The copy number of Bacteroidetes and Firmicutes were also determined and compared among the groups. Significant differences in Bacteroidetes copy number and Bact/Firm ratio among the groups were identified $(P<$ 0.002 and $P<0.001$, respectively; Table 3$)$. No significant changes in Firmicutes numbers were noted. Spearman's correlation analysis revealed a negative correlation between Bacteroidetes levels and increased BMI $(\mathrm{r}=-0.18$, $P=0.017)$. A negative correlation between Bact/Firm and BMI was also noted $(\mathrm{r}=-0.22, P=0.003)$.

Gender differences were observed in Bacteroidetes copy number in children of normal weight. Specifically, girls of a normal weight had significantly higher Bacteroidetes levels than boys of normal weight $(P<0.05$; Table 3). Further stratification of bacterial copy number by gender revealed significantly higher Bacteroidetes levels in girls of normal weight compared to obese girls $(P=0.002)$; there was no difference in Bacteroidetes levels between normal and obese boys.

\section{Discussion}

The objective of the present study was to investigate a possible correlation between the intestinal microbiota, Bacteroidetes and Firmicutes, and obesity in Kazakh school children. Significantly reduced Bacteroidetes levels as well as Bact/Firm ratio were observed in the obese group as compared to the normal weight participants, which is similar to previous reports $[4,6,9,11]$. In addition, a negative correlation between Bacteroidetes and Bact/Firm ratio with BMI was observed. These results are consistent with those reported by Ley et al. [4] in which decreased Bacteroidetes and increased Firmicutes was associated with obesity and increased energy absorption [32].

The Kazakh people are a relatively isolated minority in China and have similarities in living environment and diet, which would likely minimize the difference in the gastrointestinal microbiota among individuals. In the present study, the number of Bacteroidetes was markedly lower in obese children than in children with normal weight, resulting in a reduced Bact/Firm ratio. No difference in the Bact/Firm ratio was observed between the overweight and normal groups, which is consistent with that reported by Li et al. [9]. However, as previously stated, discrepant results have been reported. For example, in 98 subjects, including 30 with normal weight, 35 with overweight, and 33 obese individuals, the number of Bacteroidetes in overweight subjects was higher than that in the normal group. Furthermore, Duncan et al. [12] found that the number of Bacteroidetes in obese subjects was comparable with that in normal weight subjects, and the proportion of Bacteroidetes remained unchanged following diet control in obese subjects [33].

The present study also found that the difference in Bacteroidetes levels observed between the normal and obese children were mainly contributed by the values obtained in the girls as differences in Bacteroidetes levels were observed between normal and obese girls but not boys. This is different from that reported by Mueller et al. [34], who reported a higher Bacteroidetes-Prevotella number in male than in female. A result that may be explained by the age differences between these two studies. In addition, gender differences in the level of Bacteroidetes and Firmicutes were observed in those participants of

Table 3 Univariate analysis of the association of Bacteroidetes and Firmicutes with BMI levels by gender

\begin{tabular}{|c|c|c|c|c|c|}
\hline Variables & Total & Normal group & Overweight group & Obesity group & P-values \\
\hline Total & $(n=175)$ & $(n=91)$ & $(n=62)$ & $(n=22)$ & \\
\hline Bacteroidetes $\times 10^{7}$ copies $/ \mu \mathrm{L}$ & $1.31 \pm 1.94$ & $1.5 \pm 2.2$ & $1.37 \pm 1.77$ & $0.33 \pm 0.47^{\dagger}$ & $0.002^{*}$ \\
\hline Firmicutes $\times 10^{7}$ copies $/ \mu \mathrm{L}$ & $2.58 \pm 4.52$ & $2.43 \pm 4.53$ & $2.05 \pm 3.01$ & $4.7 \pm 7.01$ & 0.628 \\
\hline Bact/Firm & $0.98 \pm 0.71$ & $1.06 \pm 0.62$ & $1.03 \pm 0.82$ & $0.48 \pm 0.52^{\dagger \neq}$ & $<0.001^{*}$ \\
\hline Boy & $(n=87)$ & $(n=45)$ & $(n=30)$ & $(n=12)$ & \\
\hline Bacteroidetes $\times 10^{7}$ copies $/ \mu \mathrm{L}$ & $1.02 \pm 1.53$ & $1.00 \pm 1.42^{a}$ & $1.30 \pm 1.86$ & $0.41 \pm 0.56$ & 0.218 \\
\hline Firmicutes $\times 10^{7}$ copies $/ \mu \mathrm{L}$ & $1.99 \pm 3.38$ & $1.71 \pm 3.32^{\mathrm{a}}$ & $1.57 \pm 2.04$ & $4.12 \pm 5.36$ & 0.170 \\
\hline Bact/Firm & $1.06 \pm 0.81$ & $1.15 \pm 0.72$ & $1.12 \pm 0.97$ & $0.59 \pm 0.59$ & 0.066 \\
\hline Girl & $(n=88)$ & $(n=46)$ & $(n=32)$ & $(n=10)$ & \\
\hline Bacteroidetes $\times 10^{7}$ copies $/ \mu \mathrm{L}$ & $1.59 \pm 2.26$ & $1.99 \pm 2.69$ & $1.43 \pm 1.70$ & $0.23 \pm 0.32^{\dagger \neq}$ & $0.002^{*}$ \\
\hline Firmicutes $\times 10^{7}$ copies $/ \mu \mathrm{L}$ & $3.17 \pm 5.37$ & $3.14 \pm 5.41$ & $2.50 \pm 3.68$ & $5.39 \pm 8.87$ & 0.725 \\
\hline Bact/Firm & $0.90 \pm 0.58$ & $0.98 \pm 0.51$ & $0.94 \pm 0.66$ & $0.36 \pm 0.43^{\dagger \neq}$ & $0.003^{*}$ \\
\hline
\end{tabular}

Data were presented as mean \pm SD; Differences among three groups were compared using Kruskal-Wallis test and between two groups were compared using the Mann-Whitney $U$ test because data were not normally distributed.

${ }^{*} P<0.05$, indicated significant differences among three groups.

${ }^{t, \neq} P<0.0167$, indicated significant differences as compared with the ${ }^{\dagger}$ normal and ${ }^{\ddagger}$ overweight groups.

${ }^{a} P<0.05$, indicated significant differences between boys and girls in normal group. No significant difference between boys and girls were found either in overweight group or in obesity group. 
normal weight, but not in the overweight and obese groups, which is in agreement with Mueller et al. [34]. While the cause of this difference is unclear, gender differences in iron metabolism [35], which affects the composition of microbiota [36,37], may explain the varying levels of Bacteroidetes and Firmicutes between normal weight girls and boys observed in this study. More studies with large sample size or more populations are needed to elucidate the specific role of gastrointestinal microbiota in the pathogenesis of obesity as well as the influence of gender on microbiota composition.

Although it is known that obesity is associated with changes in composition as well as function of gut microbiota, the mechanism behind this alteration remains to be elucidated. The influence of gut microbiota on nutrient absorption and metabolism has been suggested as a possible mechanism to explain their possible relation to obesity [16]. Alternatively, altered gut microbiota may alter the exposure to obesogenic and diabetogenic environmental chemicals [38]. Furthermore, altered gut microbiota may increase proinflammatory cytokine secretion, which may be related with the low grade inflammation found in obesity and diabetes [7].

The present study has some limitations. Firstly, two main phyla of bacteria, Bacteroidetes and Firmicutes, were measured in the feces of Kazakh children; however, specific genus and species were not isolated. Schwiertz et al. [11] reported that the number of Ruminococcus flavefaciens in overweight or obese subjects was lower than that in subjects with normal weight. In addition, obese subjects had significantly reduced numbers of Clostridium leptum and Bifidobacterium. Therefore, specific genus and species will be analyzed in further studies. In addition, the limited amount of DNA obtained from the participant samples prevented the inclusion of $16 \mathrm{~S}$ sequencing, additional $\mathrm{qPCR}$ primer sets, and/or metagenomic shotgun sequencing analyses. Finally, the mechanism by which BMI influences Bacteroidetes level or vice versa was not investigated in the present study.

\section{Conclusion}

In summary, this study revealed an significant decrease in the number of Bacteroidetes in the feces of obese Kazakh girls; no significant changes in Firmicutes numbers were noted. Although the number of study subjects is greater than many previous studies, further studies with larger sample sizes are required to confirm our findings as well as identify the mechanism governing this gender difference in the regulation of intestinal microbiota.

\section{Competing interest}

The authors declare that there is no conflict of interest.

\section{Authors' contributions}

PX: guarantor of integrity of the entire study, study concepts, definition of intellectual content, manuscript review; ML: guarantor of integrity of the entire study, study design, literature research, clinical studies, data acquisition, statistical analysis, manuscript preparation, manuscript editing; JZ: clinical studies, experimental studies, data acquisition; TZ: data acquisition, data analysis. All authors read and approved the final manuscript.

\section{Acknowledgements}

This study was supported by grants from the Regional Science Foundation of the National Natural Science Foundation of China (81060072) and the General Project of Natural Science Foundation of the Xinjiang Uygur Autonomous Region (2010211A42).

\section{Author details}

'Department of Pediatrics, First Affiliated Hospital of Xinjiang Medical University, 137 Liyushan South Road, Urumqi, Xinjiang Uyghur Autonomous Region 840054, People's Republic of China. ${ }^{2}$ Xinjiang Evidence-based Medicine Research Institute, 137 Liyushan South Road, Urumqi, Xinjiang, Uyghur Autonomous Region 830054, People's Republic of China. ${ }^{3}$ Department of Urology, Second Affiliated Hospital of Xinjiang Medical University, 38 nanhu East Road, Urumqi, Xinjiang Uyghur Autonomous Region 830063, People's Republic of China.

Received: 28 March 2012 Accepted: 15 November 2012

Published: 28 November 2012

\section{References}

1. Saulnier DM, Kolida S, Gibson GR: Microbiology of the human intestinal tract and approaches for its dietary modulation. Curr Pharm Des 2009, 15:1403-1414

2. Xiong DX: Intestinal microecological preparations and the treatment of digestive tract diseases. Beijing: Science Press; 2008 (in Chinese).

3. Bäckhed F, Ley RE, Sonnenburg JL, Peterson DA, Gordon Jl: Host-bacterial mutualism in the human intestine. Science 2005, 307:1915-1920.

4. Ley RE, Peterson DA, Gordon Jl: Ecological and evolutionary forces shaping microbial diversity in the human intestine. Cell 2006, 124:837-848.

5. Ley RE, Turnbaugh PJ, Klein S, Gordon Jl: Microbial ecology: human gut microbes associated with obesity. Nature 2006, 444:1022-1023.

6. Turnbaugh PJ, Hamady M, Yatsunenko T, Cantarel BL, Duncan A, Ley RE, Sogin ML, Jones WJ, Roe BA, Affourtit JP, Egholm M, Henrissat B, Heath AC, Knight R, Gordon Jl: A core gut microbiome in obese and lean twins. Nature 2009, 457:480-484.

7. Vrieze A, Holleman F, Zoetendal EG, de Vos WM, Hoekstra JB, Nieuwdorp M: The environment within: how gut microbiota may influence metabolism and body composition. Diabetologia 2010, 53:606-613.

8. Ley RE, Bäckhed F, Turnbaugh P, Lozupone CA, Knight RD, Gordon J: Obesity alters gut microbial ecology. Proc Natl Acad Sci U S A 2005, 102:11070-11075.

9. Li M, Wang B, Zhang M, Rantalainen M, Wang S, Zhou H, Zhang Y, Shen J, Pang X, Zhang M, Wei H, Chen Y, Lu H, Zuo J, Su M, Qiu Y, Jia W, Xiao C, Smith LM, Yang S, Holmes E, Tang H, Zhao G, Nicholson JK, Li L, Zhao L: Symbiotic gut microbes modulate human metabolic phenotypes. Proc Natl Acad Sci U S A 2008, 105:2117-2122.

10. Collado MC, Isolauri E, Laitinen K, Salminen S: Distinct composition of gut microbiota during pregnancy in overweight and normal-weight women. Am J Clin Nutr 2008, 88:894-899.

11. Schwiertz A, Taras D, Schäfer K, Beijer S, Bos NA, Donus C, Hardt PD: Microbiota and SCFA in lean and overweight healthy subjects. Obesity (Silver Spring) 2010, 18:190-195.

12. Duncan SH, Lobley GE, Holtrop G, Ince J, Johnstone AM, Louis P, Flint HJ: Human colonic microbiota associated with diet, obesity and weight loss. Int J Obes (Lond) 2008, 32:1720-1724.

13. Franks AH, Harmsen HJ, Raangs GC, Jansen GJ, Schut F, Welling GW: Variations of bacterial populations in human feces measured by fluorescent in situ hybridization with group-specific 16S rRNA-targeted oligonucleotide probes. Appl Environ Microbiol 1998, 64:3336-3345.

14. Wilson $\mathrm{KH}$, Blitchington RB: Human colonic biota studied by ribosomal DNA sequence analysis. Appl Environ Microbiol 1996, 62:2273-2278. 
15. Wilson KH, Ikeda JS, Blitchington RB: Phylogenetic placement of community members of human colonic biota. Clin Infect Dis 1997, 25(Suppl 2):S114-S116

16. Bäckhed F, Ding H, Wang T, Hooper LV, Koh GY, Nagy A, Semenkovich CF, Gordon J: The gut microbiota as an environmental factor that regulates fat storage. Proc Natl Acad Sci U S A 2004, 101:15718-15723.

17. Hooper LV, Wong MH, Thelin A, Hansson L, Falk PG, Gordon Jl: Molecular analysis of commensal host-microbial relationships in the intestine. Science 2001, 291:881-884.

18. Dethlefsen L, Eckburg PB, Bik EM, Relman DA: Assembly of the human intestinal microbiota. Trends Ecol Evol 2006, 21:517-523.

19. Sears CL: A dynamic partnership: celebrating our gut flora. Anaerobe 2005, 11:247-251.

20. Tao Y, Mao X, Xie Z, Ran X, Liu X, Wang Y, Luo X, Hu M, Gen W, Zhang M, Wang T, Ren J, Wufuer H, Li L: The prevalence of type 2 diabetes and hypertension in Uygur and Kazak populations. Cardiovasc Toxicol 2008, 8:155-159.

21. Yan WL, Zheng YJ, Wu J, Chen SF, Ti XK, Li L, Liu XR: Ethnic differences in body mass index and prevalence of obesity in school children of Urumqi City, Xinjiang, China. Biomed Environ Sci 2006, 19:469-473.

22. Li M, Liu Y, Xu P, Ye M, Liu Y: Association of the rs9939609 polymorphism of FTO gene with overweight or obesity in Hazakh children. Chin J Med Genet 2010, 27:678-681. in Chinese.

23. Li M, Zhang T, Liu Y, Xu PR: The research of association between gene rs9930506 polymorphism and Hazakh children with overweight or obesity in Xinjiang. Chin J Prev Med 2010, 44:1106-1110. in Chinese.

24. Thuny F, Richet H, Casalta JP, Angelakis E, Habib G, Raoult D: Vancomycin treatment of infective endocarditis is linked with recently acquired obesity. PLoS One 2010, 5:e9074.

25. Haffner SM, Kennedy E, Gonzalez C, Stern MP, Miettinen H: A prospective analysis of the HOMA model. The Mexico city diabetes study. Diabetes Care 1996, 19:1138-1141.

26. Group of China Obesity Task Force: Body mass index reference norm for screening overweight and obesity in Chinese children and adolescents. Chin J Epidemiol 2004, 25:97-102. in Chinese.

27. Davison KK, Birch LL: Child and parent characteristics as predictors of change in girls'body mass index. Int J Obes Relat Metab Disord 2001, 25:1834-1842.

28. Lobstein T, Baur L, Uauy R: Obesity in children and young people: a crisis in public health. Obes Rev 2004, 5(Suppl 1):4-104.

29. Polley DC, Spicer MT, Knight AP, Hartley BL: Intrafamilial correlates of overweight and obesity in African-American and Native-American grandparents, parents, and children in rural Oklahoma. J Am Diet Assoc 2005, 105:262-265.

30. Salmon J, Timperio A, Telford A, Carver A, Crawford D: Association of family environment with children's television viewing and with low level of physical activity. Obes Res 2005, 13:1939-1951.

31. van der Horst K, Oenema A, Ferreira I, Wendel-Vos W, Giskes K, van Lenthe F, Brug J: A systematic review of environmental correlates of obesityrelated dietary behaviors in youth. Health Educ Res 2007, 22:203-226.

32. Jumpertz R, Le DS, Turnbaugh PJ, Trinidad C, Bogardus C, Gordon Jl, Krakoff $\mathrm{J}$ : Energy-balance studies reveal associations between gut microbes, caloric load, and nutrient absorption in humans. Am J Clin Nutr 2011, 94:58-65.

33. Duncan SH, Belenguer A, Holtrop G, Johnstone AM, Flint HJ, Lobley GE: Reduced dietary intake of carbohydrates by obese subjects results in decreased concentrations of butyrate and butyrate-producing bacteria in feces. Appl Environ Microbio/ 2007, 73:1073-1078.

34. Mueller S, Saunier K, Hanisch C, Norin E, Alm L, Midtvedt T, Cresci A, Silvi S, Orpianesi C, Verdenelli MC, Clavel T, Koebnick C, Zunft HJ, Doré J, Blaut M: Differences in fecal microbiota in different European study populations in relation to age, gender, and country: a cross-sectional study. Appl Environ Microbiol 2006, 72:1027-1033.

35. Harrison-Findik DD: Gender-related variations in iron metabolism and liver diseases. World J Hepatol 2010, 2:302-310.

36. Buhnik-Rosenblau K, Moshe-Belizowski S, Danin-Poleg Y, Meyron-Holtz EG: Genetic modification of iron metabolism in mice affects the gut microbiota. Biometals 2012, 25:883-892.
37. Tompkins GR, O'Dell NL, Bryson IT, Pennington CB: The effects of dietary ferric iron and iron deprivation on the bacterial composition of the mouse intestine. Curr Microbiol 2001, 43:38-42.

38. Snedeker SM, Hay AG: Do interactions between gut ecology and environmental chemicals contribute to obesity and diabetes? Environ Health Perspect 2012, 120:332-339.

doi:10.1186/1471-2180-12-283

Cite this article as: Xu et al.: Correlation of intestinal microbiota with overweight and obesity in Kazakh school children. BMC Microbiology 2012 12:283.

\section{Submit your next manuscript to BioMed Central and take full advantage of:}

- Convenient online submission

- Thorough peer review

- No space constraints or color figure charges

- Immediate publication on acceptance

- Inclusion in PubMed, CAS, Scopus and Google Scholar

- Research which is freely available for redistribution 